\title{
Fault-Tolerant Control Based on Virtual Actuator and Sensor for Discrete-Time Descriptor Systems
}

\author{
Ye Wang ${ }^{(\mathbb{D}}$, Damiano Rotondo, Vicenç Puig ${ }^{(}$, and Gabriela Cembrano ${ }^{(}$
}

\begin{abstract}
This article proposes a fault-tolerant control (FTC) strategy based on virtual actuator and sensor for discrete-time descriptor systems subject to actuator and sensor faults. The fault-tolerant closed-loop system, which includes the nominal controller and observer, as well as the virtual actuator and the virtual sensor, hides the effects of faults. When an observer-based state-feedback law is considered, the existence of algebraic loop may prevent the practical implementation due to the current algebraic states depending on the current control input, that affects also the implementation of the virtual actuator/sensor. To deal with this issue, an observer-based delayed feedback controller and a delayed virtual actuator are proposed for discrete-time descriptor systems. Furthermore, the satisfaction of the separation principle is shown, and an improved admissibility condition is developed for the design of the controller and virtual actuator/sensor. Finally, some simulation results including an electrical circuit are used to demonstrate the applicability of the proposed methods.
\end{abstract}

Index Terms-Fault-tolerant control, virtual actuator, virtual sensor, improved admissibility condition, descriptor systems.

\section{INTRODUCTION}

D ESCRIPTOR systems [1], also known as singular systems [2] or differential-algebraic systems [3], are described by both differential and algebraic equations, so that they provide a more natural representation of physical systems comprising static and dynamic behavior. In simple terms,

Manuscript received January 30, 2020; revised June 25, 2020 and July 27, 2020; accepted August 8, 2020. Date of publication August 19, 2020; date of current version December 1, 2020. This work was supported by the Fundamental Research Funds for the Central University under Grant 3072020CFJ040. This work was also partially funded by the Spanish State Research Agency (AEI) and the European Regional Development Fund (ERFD) through the project SCAV (ref. MINECO DPI2017-88403-R), by the DGR of Generalitat de Catalunya (SAC group ref. 2017/SGR/482) and by SMART Project (ref. num. EFA153/16 Interreg Cooperation Program POCTEFA 2014-2020). This article was recommended by Associate Editor A. James. (Corresponding author: Ye Wang.)

Ye Wang is with the College of Intelligent Systems Science and Engineering, Harbin Engineering University, Harbin 150001, China, also with the Engineering Research Center of Navigation Instruments, Ministry of Education, Harbin 150001, China, and also with the Department of Electrical and Electronic Engineering, University of Melbourne, Melbourne, VIC 3010, Australia (e-mail: ye.wang@outlook.es).

Damiano Rotondo is with the Department of Electrical Engineering and Computer Science (IDE), University of Stavanger, 4009 Stavanger, Norway (e-mail: damiano.rotondo@uis.no).

Vicenç Puig is with the Automatic Control Department, Institut de Robòtica i Informàtica Industrial (IRI), CSIC-UPC, Universitat Politècnica de Catalunya-BarcelonaTech (UPC), 08028 Barcelona, Spain (e-mail: vicenc.puig@upc.edu).

Gabriela Cembrano is with the Automatic Control Department, Institut de Robòtica i Informàtica Industrial (IRI), CSIC-UPC, Universitat Politècnica de Catalunya-BarcelonaTech (UPC), 08028 Barcelona, Spain, and also with the CETaqua, Water Technology Centre, 08940 Barcelona, Spain (e-mail: cembrano@iri.upc.edu).

Color versions of one or more of the figures in this article are available online at https://ieeexplore.ieee.org.

Digital Object Identifier 10.1109/TCSI.2020.3015887 the main difference between descriptor and dynamic systems is that in the former the derivative-term matrix may be singular whereas in the latter it is an identity matrix. Although their analysis is somehow more complicated, since it is necessary to ensure not only stability, but also the existence of a unique solution and the causality (a set of properties commonly referred to as admissibility), their superiority in describing real-world systems has led to their applications in a very wide range of fields, such as economics [4], biological systems [5], water distribution networks [6], flight vehicle control [7], and robotics [8], among others.

\section{A. Motivation}

Due to the increasing complexity of modern engineering systems, the possibility of actuator and sensor malfunctioning/faults has increased dramatically. These faults may degrade the performance, leading to unsatisfactory behavior, or in the worst-case to instability, thus bearing catastrophic consequences for the system itself and for the safety of living beings around them. Motivated by the increasing need for safety and reliability, fault diagnosis and fault tolerant control (FTC) techniques have attracted a lot of interest in the control community, since they allow to maintain the system performance close to the desired one while preserving stability in spite of the faults [9]-[11]. Following a well-established nomenclature, the existing FTC techniques can be classified into passive and active [12]. In passive FTC, faults are considered as uncertainties and the controller is designed using robust control, leading to simpler implementation but more conservativeness in terms of performance and fault-tolerance [13]. On the other hand, active FTC reacts to faults by performing some reconfiguration action based on online information coming from a fault diagnosis module [14]. Recently, a few works have considered fault diagnosis [15], [16] and FTC [17]-[19] for descriptor systems. However, these techniques require an online modification of the controller/observer so that in presence of an already designed and operating control system, fault tolerance can be enforced only by means of redesigning from the scratch its components. The development of new FTC techniques that do not require such a redesign, but can be applied to existing control systems in a plug-and-play fashion, would allow for a more extensive enablement of fault tolerance in descriptor control systems.

\section{B. State-of-the-Art}

Among the successful active FTC paradigms proposed to achieve fault tolerance, there is fault-hiding [20], which reconfigures the faulty plant so that from the point of view of the 


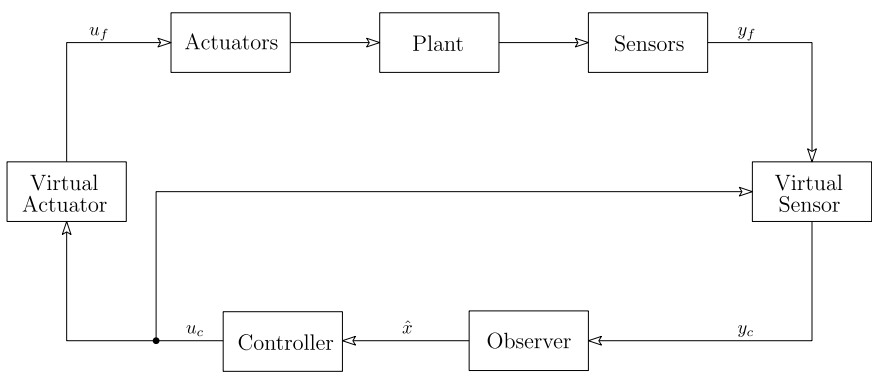

Fig. 1. Active FTC Scheme using VA and VS.

controller/observer no fault is perceived, in such a way that these components can be kept in the loop without modifications. Fault-hiding is achieved by inserting appropriate blocks in the loop, which are called virtual actuators (VA) in the case of actuator faults and virtual sensors (VS) in the case of sensor faults. The main advantage of this approach with respect to other existing alternatives is that fault tolerance can be added to an already existing control scheme following a plug-and-play philosophy [21]. This strategy has been applied successfully to several classes of systems, such as linear systems [22], piecewise affine [23], Hammerstein-Wiener [24], Takagi-Sugeno [25] and linear parameter varying [26], [27]. Furthermore, some recent research has integrated virtual actuators with a switched formulation to achieve secure control against denial of service attacks [28]. However, to the best of the authors' knowledge, no formulation of virtual actuators/sensors is available for descriptor systems.

\section{Contributions}

This work proposes an active FTC strategy using VA and VS technique to the case of descriptor systems. In particular, the contributions of this article are summarized as follows:

- The two techniques are merged together in a formulation that allows handling at the same time partial and even total losses of actuators/sensors, as well as additive faults. It is worth noting that the overall FTC scheme, presented in Figure 1, includes a state observer.

- Notably, so far, the problem of observer-based statefeedback control has been addressed only in the case of continuous-time systems [29], but not in the discrete-time case. This is due to the appearance of an algebraic loop in the implementation, which prevents the use of a classical state-feedback control law. In order to break this algebraic loop, we propose a delayed observer-based state-feedback control law. The closed-loop system arising from this choice can be modeled as a descriptor system with state delay. We show that for the augmented system, composed of the system itself, the controller and the state observer, a separation principle holds, which allows the separate design of each component.

- This property is later used to show that also the virtual actuator, which is also delayed, and the virtual sensor can be designed separately. Finally, a new admissibility condition that decreases conservativeness with respect to existing results is proposed to design the delayed state-feedback and virtual actuator gains, while the observer and virtual sensor design is performed based on Lyapunov stability.

\section{Outline}

The remainder of this article is structured as follows. Section II provides a brief statement of the problem of interest. Section III discusses the observer-based feedback control of discrete-time descriptor systems by motivating the use of a delayed control action, showing the existence of a separation principle, and proposing an improved admissibility condition that can be used for the design of the delayed state-feedback gain. Section IV describes the extension of the virtual actuator and virtual sensor to the case of descriptor systems. Simulation results are shown in Section $\mathrm{V}$ to validate the theoretical results. Finally, some conclusions are outlined in Section VI.

\section{E. Notation}

We use $I$ to denote an identity matrix of appropriate dimension. For a matrix $X$, we use $X^{\top}, X^{\dagger}$ and $\operatorname{rank}(X)$ to denote the transpose, the pseudo inverse and the rank of $X$. $\operatorname{He}(X)=X+X^{\top}$. We use $X \succ 0(X \prec 0)$ to denote that the matrix $X$ is positive (negative) definite. We use $X \succeq 0$ to denote that the matrix $X$ is positive semi-definite. We also define the following sets: $\mathbb{S}^{n}:=\left\{X \in \mathbb{R}^{n \times n} \mid X=X^{\top}\right\}$, $\mathbb{S}_{\succ 0}^{n}:=\quad\left\{X \in \mathbb{R}^{n \times n} \mid X=X^{\top}, X \succ 0\right\}$ and $\mathbb{S}_{\geq 0}^{n}:=$ $\left\{X \in \mathbb{R}^{n \times n} \mid X=X^{\top}, X \succeq 0\right\}$. For two matrices $X$ and $Y$, we use $\operatorname{diag}(X, Y)$ to denote a block diagonal matrix with elements defined by $X$ and $Y$. For a singular matrix $M \in \mathbb{R}^{n \times n}$ with $\operatorname{rank}(M)=r$ and $r<n$, let $M^{\perp} \in \mathbb{R}^{(n-r) \times n}$ be any matrix such that $M^{\perp} M=0$ and $M^{\perp} M^{\perp^{\top}} \succ 0$.

\section{Problem Statement}

Consider the following discrete-time descriptor system subject to actuator and sensor faults

$$
\begin{aligned}
E x_{f}(k+1) & =A x_{f}(k)+B f(\phi(k))\left(u_{f}(k)+f_{a}(k)\right) \\
y_{f}(k) & =C_{f}(\gamma(k)) x_{f}(k)+f_{s}(k),
\end{aligned}
$$

where $x_{f} \in \mathbb{R}^{n_{x}}, u_{f} \in \mathbb{R}^{n_{u}}$ and $y_{f} \in \mathbb{R}^{n_{y}}$ denote the vectors of faulty system states, faulty control inputs and faulty measurement output vectors, respectively. $f_{a} \in \mathbb{R}^{n_{u}}$ and $f_{s} \in$ $\mathbb{R}^{n_{y}}$ denote the vectors of additive actuator and sensor faults. $\phi \in \mathbb{R}^{n_{u}}$ and $\gamma \in \mathbb{R}^{n_{y}}$ denote the vectors of multiplicative actuator and sensor faults with

$\phi(k)=\left[\phi_{1}(k), \ldots, \phi_{n_{u}}(k)\right]^{\top}, 0 \leq \phi_{i}(k) \leq 1, i=1, \ldots, n_{u}$,
$\gamma(k)=\left[\gamma_{1}(k), \ldots, \gamma_{n_{y}}(k)\right]^{\top}, 0 \leq \gamma_{j}(k) \leq 1, j=1, \ldots, n_{y}$,
Besides, $A \in \mathbb{R}^{n_{x} \times n_{x}}$ and $B_{f}(\phi(k)) \in \mathbb{R}^{n_{x} \times n_{u}}$ and $C_{f}(\gamma(k)) \in \mathbb{R}^{n_{y} \times n_{x}}$ are defined as follows:

$$
\begin{aligned}
& B_{f}(\phi(k))=B \operatorname{diag}\left(\phi_{1}(k), \ldots, \phi_{n_{u}}(k)\right), \\
& C_{f}(\gamma(k))=\operatorname{diag}\left(\gamma_{1}(k), \ldots, \gamma_{n_{y}}(k)\right) C,
\end{aligned}
$$

where $B$ and $C$ are given in the nominal descriptor system (3).

Assumption 1: The additive and multiplicative actuator and sensor faults are assumed to be estimated as known variables. 


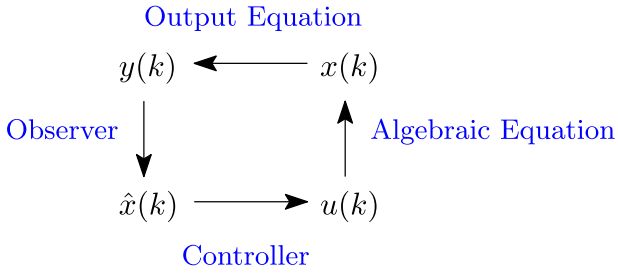

Fig. 2. Algebraic Loop in Implementation.

As described in Section I, and depicted in Figure 1, the ultimate goal of this article is to design a VA and a VS for the reconfiguration of the faulty system (1), so as to achieve fault tolerance.

\section{Observer-Based Feedback Controller FOR DESCRIPTOR SYSTEMS}

In this section, we first discuss an observer-based feedback control of the nominal system (without faults). Let us consider the following discrete-time descriptor system, which corresponds to (1) under no faults

$$
\begin{aligned}
E x(k+1) & =A x(k)+B u(k), \\
y(k) & =C x(k),
\end{aligned}
$$

where $x \in \mathbb{R}^{n}, u \in \mathbb{R}^{m}$ and $y \in \mathbb{R}^{p}$ denote the system state, control input and measurement output vectors, respectively. $A \in \mathbb{R}^{n \times n}, B \in \mathbb{R}^{n \times m}, C \in \mathbb{R}^{p \times n}$ and $E \in \mathbb{R}^{n \times n}$ are state-space matrices, with $E$ possibly singular, such that $\operatorname{rank}(E)=r \leq n$.

Under the assumption that the descriptor system (3) is observable and hence matrices $E$ and $C$ satisfy $\operatorname{rank}\left(\left[\begin{array}{ll}E^{\top} & C^{\top}\end{array}\right]\right)=n$, it is possible to use the observer structure from [30]

$$
\begin{aligned}
z(k+1) & =(T A-L C) \hat{x}(k)+T B u(k)+L y(k), \\
\hat{x}(k) & =z(k)+N y(k),
\end{aligned}
$$

where $z \in \mathbb{R}^{n}$ and $\hat{x} \in \mathbb{R}^{n}$ denote the observer state and the estimated state, respectively, $L \in \mathbb{R}^{n \times p}$ is an observer gain. Besides, $T \in \mathbb{R}^{n \times n}$ and $N \in \mathbb{R}^{n \times p}$ are matrices such that

$$
T E+N C=I,
$$

whose existence is guaranteed by the above-mentioned observability and rank conditions.

To make the descriptor system (3) admissible, i.e. regular, causal and stable, using a feedback law fed by the estimated state $\hat{x}$, one might propose to use a standard state-feedback law, $u(k)=K \hat{x}(k)$, where $K \in \mathbb{R}^{n \times m}$ is a controller gain. However, in many cases, the choice $u(k)=K \hat{x}(k)$ creates an algebraic loop, which makes the practical implementation impossible as shown in Figure 2. In fact, $u(k)$ depends on $\hat{x}(k)$, which is calculated using $y(k)$ through (4b). The output vector $y(k)$ depends on $x(k)$ through (3b), which might depend on $u(k)$ itself if $\operatorname{rank}\left(\left[\begin{array}{ll}E & B\end{array}\right]\right) \neq \operatorname{rank}(E)$.

For this reason, in this article we propose a delayed state feedback to perform the observer-based control of the descriptor system (3), as follows:

$$
u(k)=K \hat{x}(k-1) .
$$

Thus, the closed-loop system can be modeled as a descriptor system with state delay. In the remainder of the section, we discuss about this choice by showing that the separation principle still holds, so that the controller and the observer design can be performed independently. Then, by revisiting some preliminary results for discrete-time descriptor systems with state delay given in [2], we propose an improved admissibility condition for these systems taking into account a Lyapunov functional in [31], which is later used to present a design procedure for the observer-based state-feedback control of discrete-time descriptor systems.

\section{A. Separation Principle}

Define the state estimation error $e(k)=x(k)-\hat{x}(k)$. From (3b) and (4b) with (5), this error dynamics can be formulated as $e(k+1)=(T A-L C) e(k)$. Besides, using (6) with $\hat{x}(k-1)=x(k-1)-e(k-1)$, the descriptor system (3) can be rewritten as $\operatorname{Ex}(k+1)=A x(k)+B K x(k-1)-$ $B K e(k-1)$.

As a result, the augmented system can be expressed as the descriptor system with state delay

$$
\bar{E}\left[\begin{array}{l}
x(k+1) \\
e(k+1)
\end{array}\right]=\bar{A}\left[\begin{array}{l}
x(k) \\
e(k)
\end{array}\right]+\bar{A}_{d}\left[\begin{array}{l}
x(k-1) \\
e(k-1)
\end{array}\right],
$$

with

$$
\bar{E}=\left[\begin{array}{ll}
E & 0 \\
0 & I
\end{array}\right], \bar{A}=\left[\begin{array}{cc}
A & 0 \\
0 & (T A-L C)
\end{array}\right], \bar{A}_{d}=\left[\begin{array}{cc}
B K & -B K \\
0 & 0
\end{array}\right] .
$$

Let us consider the class of discrete-time descriptor systems with state delay

$$
E x(k+1)=A x(k)+A_{d} x(k-1) .
$$

According to [2, pp. 178], we first recall the following definition.

Definition 1: The discrete-time descriptor system with state delay (8) is said to be

- regular if $\operatorname{det}\left(z^{2} E-z A-A_{d}\right)$ is not identically zero;

- causal if it is regular and

$$
\operatorname{deg}\left(z^{n} \operatorname{det}\left(z E-A-z^{-1} A_{d}\right)\right)=n+\operatorname{rank}(E) ;
$$

- stable if it is regular and $\max (|v|)<1$, with $v \in$ $\lambda\left(E, A, A_{d}\right)$, where

$$
\lambda\left(E, A, A_{d}\right)=\left\{z: \operatorname{det}\left(z^{2} E-z A-A_{d}\right)=0\right\} ;
$$

- admissible if it is regular, causal and stable.

The following theorem establishes the separation principle for a discrete-time descriptor system with state delay in a block-triangular form, i.e. (7).

Theorem 1: The following statements are equivalent:

- The descriptor systems with state delay

$$
\begin{aligned}
& E_{1} x_{1}(k+1)=A_{11} x_{1}(k)+A_{d_{11}} x_{1}(k-1), \\
& E_{2} x_{2}(k+1)=A_{22} x_{2}(k)+A_{d_{22}} x_{2}(k-1),
\end{aligned}
$$

with $x_{1} \in \mathbb{R}^{n_{1}}$ and $x_{2} \in \mathbb{R}^{n_{2}}$ are admissible. 
- The descriptor system with state delay

$$
\begin{gathered}
{\left[\begin{array}{cc}
E_{1} & 0 \\
0 & E_{2}
\end{array}\right]\left[\begin{array}{l}
x_{1}(k+1) \\
x_{2}(k+1)
\end{array}\right]=\left[\begin{array}{cc}
A_{11} & A_{12} \\
0 & A_{22}
\end{array}\right]\left[\begin{array}{l}
x_{1}(k) \\
x_{2}(k)
\end{array}\right]} \\
+\left[\begin{array}{cc}
A_{d_{11}} & A_{d_{12}} \\
0 & A_{d_{22}}
\end{array}\right]\left[\begin{array}{l}
x_{1}(k-1) \\
x_{2}(k-1)
\end{array}\right],
\end{gathered}
$$

with $A_{12} \in \mathbb{R}^{n_{1} \times n_{2}}$ and $A_{d_{12}} \in \mathbb{R}^{n_{1} \times n_{2}}$ is admissible.

Proof: (Regularity) The following equality holds

$$
\begin{aligned}
& \operatorname{det}\left(z^{2}\left[\begin{array}{cc}
E_{1} & 0 \\
0 & E_{2}
\end{array}\right]-z\left[\begin{array}{cc}
A_{11} & A_{12} \\
0 & A_{22}
\end{array}\right]-\left[\begin{array}{cc}
A_{d 11} & A_{d 12} \\
0 & A_{d 22}
\end{array}\right]\right) \\
& =\operatorname{det}\left(\left[\begin{array}{cc}
z^{2} E_{1}-z A_{11}-A_{d 11} & -z A_{12}-A_{d 12} \\
0 & z^{2} E_{2}-z A_{22}-A_{d 22}
\end{array}\right]\right) \\
& =\operatorname{det}\left(z^{2} E_{1}-z A_{11}-A_{d 11}\right) \operatorname{det}\left(z^{2} E_{2}-z A_{22}-A_{d 22}\right) .
\end{aligned}
$$

Hence, according to Definition 1, the regularity of the systems (9a) and (9b) is equivalent to that of the system (10).

(Causality) Let us denote

$$
\Psi=\left[\begin{array}{cc}
z E_{1}-A_{11}-z^{-1} A_{d 11} & -A_{12}-z^{-1} A_{d 12} \\
0 & z E_{2}-A_{22}-z^{-1} A_{d 22}
\end{array}\right] .
$$

According to Definition 1, we also have that

$$
\begin{aligned}
\operatorname{deg} & \left(z^{n_{1}+n_{2}} \operatorname{det}(\Psi)\right) \\
= & \operatorname{deg}\left(z^{n_{1}} \operatorname{det}\left(z E_{1}-A_{11}-z^{-1} A_{d 11}\right)\right. \\
& \left.\times z^{n_{2}} \operatorname{det}\left(z E_{2}-A_{22}-z^{-1} A_{d 22}\right)\right) \\
= & \operatorname{deg}\left(z^{n_{1}} \operatorname{det}\left(z E_{1}-A_{11}-z^{-1} A_{d 11}\right)\right) \\
& +\operatorname{deg}\left(z^{n_{2}} \operatorname{det}\left(z E_{2}-A_{22}-z^{-1} A_{d 22}\right)\right) .
\end{aligned}
$$

From causality of the systems (9a) and (9b), it follows that $\operatorname{deg}\left(z^{n_{1}} \operatorname{det}\left(z E_{1}-A_{11}-z^{-1} A_{d 11}\right)\right)=n_{1}+\operatorname{rank}\left(E_{1}\right)$ and $\operatorname{deg}\left(z^{n_{2}} \operatorname{det}\left(z E_{2}-A_{22}-z^{-1} A_{d 22}\right)\right)=n_{2}+\operatorname{rank}\left(E_{2}\right)$. Then, we know that $\operatorname{deg}\left(z^{n_{1}+n_{2}} \operatorname{det}(\Psi)\right)=n_{1}+\operatorname{rank}\left(E_{1}\right)+n_{2}+$ $\operatorname{rank}\left(E_{2}\right)=\left(n_{1}+n_{2}\right)+\operatorname{rank}\left(\begin{array}{cc}E_{1} & 0 \\ 0 & E_{2}\end{array}\right)$, which implies causality of the system (10).

On the other hand, for the pairs $\left(E_{1}, A_{11}, A_{d_{11}}\right)$ and $\left(E_{2}, A_{22}, A_{d_{22}}\right)$, we have that

$$
\begin{aligned}
& \operatorname{deg}\left(z^{n_{1}} \operatorname{det}\left(z E_{1}-A_{11}-z^{-1} A_{d 11}\right)\right) \leq n_{1}+\operatorname{rank}\left(E_{1}\right), \\
& \operatorname{deg}\left(z^{n_{2}} \operatorname{det}\left(z E_{2}-A_{22}-z^{-1} A_{d 22}\right)\right) \leq n_{2}+\operatorname{rank}\left(E_{2}\right) .
\end{aligned}
$$

From causality of the system (10), it follows

$$
\operatorname{deg}\left(z^{n_{1}+n_{2}} \operatorname{det}(\Psi)\right)=\left(n_{1}+n_{2}\right)+\operatorname{rank}\left(E_{1}\right)+\operatorname{rank}\left(E_{2}\right),
$$

which implies $\operatorname{deg}\left(z^{n_{1}} \operatorname{det}\left(z E_{1}-A_{11}-z^{-1} A_{d 11}\right)\right)=n_{1}+$ $\operatorname{rank}\left(E_{1}\right)$ and $\operatorname{deg}\left(z^{n_{2}} \operatorname{det}\left(z E_{2}-A_{22}-z^{-1} A_{d 22}\right)\right)=n_{2}+$ $\operatorname{rank}\left(E_{2}\right)$, and therefore causality of the systems (9a) and (9b).

(Stability) Following the proof of regularity, we also have that $\lambda\left(\begin{array}{cccccc}E_{1} & 0 \\ 0 & E_{2} & A_{11} & A_{12} & A_{d_{11}} & A_{d_{12}} \\ 0 & A_{22}, & 0 & A_{d_{22}}\end{array}\right)=\lambda\left(E_{1}, A_{1}, A_{d 11}\right) \cup$ $\lambda\left(E_{2}, A_{2}, A_{d 22}\right)$, which implies the equivalence of the stability according to Definition 1.

(Admissibility) Since we have proved the equivalence of regularity, causality and stability in systems (9a), (9b) and(10), we can conclude the equivalence of admissibility of systems (9a) and (9b) and the system (10).
Theorem 1 is crucial, since it states that the admissibility of (7) can be enforced by considering independently the systems

$$
\begin{aligned}
E x(k+1) & =A x(k)+B K x(k-1), \\
e(k+1) & =(T A-L C) e(k),
\end{aligned}
$$

where (11b) is in a dynamical form without state delay so that the design of a stabilizing observer gain $L$ can be performed using results widely available in the literature. For this reason, hereafter we focus on the design of a delayed controller gain $K$ that guarantees the admissibility of (11a). First, we present an improved admissibility condition for the descriptor system with state delay (8). Then, we propose the design condition of the delayed controller using matrix inequalities.

\section{B. Improved Admissibility Condition}

Let us recall [2, Theorem 9.3], which provides a sufficient condition for the admissibility of the system (8).

Proposition 1: The discrete-time descriptor system with state delay (8) is admissible if there exist matrices $P \in \mathbb{S}^{n}$ and $Q \in \mathbb{S}_{\succeq 0}^{n}$ such that

$$
\begin{aligned}
E^{\top} P E & \succeq 0, \\
{\left[\begin{array}{cc}
A^{\top} P A-E^{\top} P E+Q & A^{\top} P A_{d} \\
A_{d}^{\top} P A & A_{d}^{\top} P A_{d}-Q
\end{array}\right] } & \prec 0 .
\end{aligned}
$$

Proof: Based on [2, Theorem 9.3] with $\tau=1$, we can obtain (12) with $Q \succeq 0$. Since $\tau=1$, following the proof of [2, Theorem 9.3], we know the matrix $\hat{P}=\operatorname{diag}(P, Q)$ and $Q$ does not appear in [2, Eq. (9.33)] and hence can be positive semi-definite.

However, as stated by [31], conditions as the ones provided by Proposition 1 may lead to conservativeness, since the considered Lyapunov functional is of the type

$$
V(k)=x(k)^{\top} E^{\top} P E x(k)+x(k-1)^{\top} Q x(k-1),
$$

and the possibility of introducing an additional term related to $(x(k)-x(k-1))$ is ignored. Inspired by the choice of the Lyapunov functional in [31, Eq. (6)], we now present an improved admissibility condition in the following theorem.

Theorem 2: The discrete-time descriptor system with state delay (8) is admissible if there exist matrices $P \in \mathbb{S}^{n}, Q \in \mathbb{S}^{n}$ and $S \in \mathbb{S}^{n}$ such that

$$
\begin{aligned}
{\left[\begin{array}{cc}
E^{\top}(P+S) E & -E^{\top} S E \\
-E^{\top} S E & Q+E^{\top} S E
\end{array}\right] } & \succeq 0, \\
{\left[\begin{array}{ll}
\phi_{1} & \phi_{2} \\
\phi_{2}^{\top} & \phi_{3}
\end{array}\right] } & \prec 0,
\end{aligned}
$$

with

$$
\begin{aligned}
& \phi_{1}=A^{\top}(P+S) A+Q-E^{\top} P E-\mathbf{H e}\left(E^{\top} S A\right) \\
& \phi_{2}=A^{\top}(P+S) A_{d}-E^{\top} S A_{d}+E^{\top} S E \\
& \phi_{3}=A_{d}^{\top}(P+S) A_{d}-Q-E^{\top} S E .
\end{aligned}
$$

Proof: Define the variable $\xi(k)=\left[x(k)^{\top} x(k-1)^{\top}\right]^{\top}$. Then, it can be verified that the system (8) can be rewritten as

$$
\hat{E} \xi(k+1)=\hat{A} \xi(k),
$$


where

$$
\hat{E}=\left[\begin{array}{ll}
E & 0 \\
0 & I
\end{array}\right], \hat{A}=\left[\begin{array}{cc}
A & A_{d} \\
I & 0
\end{array}\right] .
$$

Set

$$
\hat{P}=\left[\begin{array}{cc}
P+S & -S E \\
-E^{\top} S & Q+E^{\top} S E
\end{array}\right] .
$$

For the system (15), it can be shown that

$$
\hat{A}^{\top} \hat{P} \hat{A}-\hat{E}^{\top} \hat{P} \hat{E}=\left[\begin{array}{ll}
\phi_{1} & \phi_{2} \\
\phi_{2}^{\top} & \phi_{3}
\end{array}\right] \prec 0 .
$$

By noting that $\hat{E}^{\top} \hat{P} \hat{E} \succeq 0$ due to (13a), and employing [32, Theorem 2], we have that the system (15) is admissible. From regularity of (15), it follows that $\operatorname{det}(z \hat{E}-\hat{A})$ is not identically zero, and since $\operatorname{det}(z \hat{E}-\hat{A})=\operatorname{det}\left(z^{2} E-z A-A_{d}\right)$, regularity of (8) follows from Definition 1 . Moreover, from the causality of (15), we have that $\operatorname{deg}(\operatorname{det}(z \hat{E}-\hat{A}))=\operatorname{rank}(\hat{E})=$ $n+\operatorname{rank}(E)$, which proves causality of $(8) \operatorname{since} \operatorname{det}(z \hat{E}-\hat{A})=$ $z^{n} \operatorname{det}\left(z E-A-z^{-1} A_{d}\right)$. Finally, the stability of (15) implies the stability of (8) and, therefore, its admissibility.

Remark 1: Note that Theorem 2 can be reduced to Proposition 1 when $S=0$.

The condition of Theorem 2 includes non-strict inequalities due to (13a). Following the spirit of [2, Theorem 9.4], we next present the admissibility condition with strict inequalities.

Theorem 3: The discrete-time descriptor system with state delay (8) is admissible if there exist matrices $P \in \mathbb{S}^{n}, Q \in \mathbb{S}^{n}$, $S \in \mathbb{S}^{n}$ and $W \in \mathbb{R}^{2 n \times(n-r)}$ such that

$$
\begin{aligned}
\hat{P} & \succ 0, \\
{\left[\begin{array}{ll}
\phi_{1} & \phi_{2} \\
\phi_{2}^{\top} & \phi_{3}
\end{array}\right]+\mathbf{H e}\left(W E^{\perp}\left[\begin{array}{ll}
A & A_{d}
\end{array}\right]\right) } & \prec 0,
\end{aligned}
$$

with $\hat{P}$ as in (17), and $\phi_{1}, \phi_{2}$ and $\phi_{3}$ as in (14).

Proof: Consider the matrix $\hat{E}^{\perp}=\left[\begin{array}{ll}E^{\perp} & 0\end{array}\right]$, which is of full row rank and satisfies $\hat{E}^{\perp} \hat{E}=0$ with $\hat{E}$ defined as in (16). From (18), we have that

$$
\begin{aligned}
\hat{A}^{\top} \hat{P} \hat{A}-\hat{E}^{\top} \hat{P} \hat{E}+\mathbf{H e}\left(W \hat{E}^{\perp} \hat{A}\right) \\
\quad=\left[\begin{array}{ll}
\phi_{1} & \phi_{2} \\
\phi_{2}^{\top} & \phi_{3}
\end{array}\right]+\mathbf{H e}\left(W E^{\perp}\left[\begin{array}{ll}
A & A_{d}
\end{array}\right]\right) \prec 0 .
\end{aligned}
$$

Since $\hat{P} \succ 0$, according to [33, Theorem 1], the system (15) is admissible. Following the proof of Theorem 2, the system (8) is shown to be admissible.

Remark 2: Note that Theorem 3 can be also reduced to [2, Theorem 9.4] when $S=0$.

\section{Delayed Controller Synthesis}

Based on above results, we now present the condition for the design of a delayed controller gain $K$ in (6), which is obtained by applying Theorem 3 taking into account that (11a) is in the form of (8) with $A_{d}=B K$.

Theorem 4: The discrete-time descriptor system with state delay (11a) is admissible if there exist matrices $P \in \mathbb{S}^{n}$,
$Q \in \mathbb{S}^{n}, S \in \mathbb{S}^{n}, W_{1} \in \mathbb{R}^{n \times(n-r)}, W_{2} \in \mathbb{R}^{n \times(n-r)}$ and $K \in \mathbb{R}^{m \times n}$ such that (19a) and

$$
\left[\begin{array}{ccc}
\psi_{1} & \psi_{2} & A^{\top}(P+S) \\
\psi_{2}^{\top} & \psi_{3} & K^{\top} B^{\top}(P+S) \\
(P+S) A & (P+S) B K & -(P+S)
\end{array}\right] \prec 0
$$

with $\hat{P}$ as in (17) and

$$
\begin{aligned}
& \psi_{1}=Q-E^{\top} P E+\mathbf{H e}\left(W_{1} E^{\perp} A-E^{\top} S A\right), \\
& \psi_{2}=W_{1} E^{\perp} B K+A^{\top}\left(E^{\perp}\right)^{\top} W_{2}^{\top}-E^{\top} S B K+E^{\top} S E, \\
& \psi_{3}=\mathbf{H e}\left(W_{2} E^{\perp} B K\right)-Q-E^{\top} S E .
\end{aligned}
$$

Proof: According to (19b), let us set $W=\left[\begin{array}{ll}W_{1}^{\top} & W_{2}^{\top}\end{array}\right]^{\top}$ and $A_{d}=B K$. Taking into account that the positive definiteness of the matrix $(P+S)$ is ensured by (19a), applying the Schur complement to (19b), we obtain

$$
\left[\begin{array}{ccc}
\psi_{1} & \psi_{2} & A^{\top} \\
\psi_{2}^{\top} & \psi_{3} & K^{\top} B^{\top} \\
A & B K & -(P+S)^{-1}
\end{array}\right] \prec 0 .
$$

By pre- and post-multiplying the above inequality by $\operatorname{diag}(I, P+S)$, we thus obtain $(20)$.

\section{Fault-Tolerant Control Based on Virtual ACTUATOR AND SENSOR FOR DESCRIPTOR Systems}

In this section, we present an FTC strategy for discrete-time descriptor systems. Taking into account the structure of the delayed feedback controller in Section III, a delayed virtual actuator and a virtual sensor for faulty descriptor systems are defined, with the goal of achieving fault tolerance by hiding the effects of faults in the closed-loop system.

\section{A. Nominal Observer-Based Delayed Controller}

For the remaining of the section, the observer-based delayed state-feedback controller in (4) and (6) will be referred to as the nominal controller/observer:

$$
\begin{aligned}
u_{c}(k) & =K \hat{x}(k-1), \\
z(k+1) & =(T A-L C) \hat{x}(k)+T B u_{c}(k)+L y_{c}(k), \\
\hat{x}(k) & =z(k)+N y_{c}(k),
\end{aligned}
$$

where $u_{c} \in \mathbb{R}^{n_{u}}$ and $y_{c} \in \mathbb{R}^{n_{y}}$ are the nominal input and output vectors, respectively.

\section{B. Delayed Virtual Actuator and Virtual Sensor for Descriptor Systems}

In order to define the delayed virtual actuator, let us consider the following matrices:

$$
\begin{aligned}
N_{v a}(\phi(k)) & =B_{f}(\phi(k))^{\dagger} B, \\
B^{*} & =B_{f}(\phi(k)) N_{v a}(\phi(k)) .
\end{aligned}
$$


Definition 2 Delayed virtual actuator for descriptor systems: Given the descriptor system subject to actuator and sensor faults in (1), the delayed virtual actuator is given by:

$$
\begin{aligned}
E x_{v a}(k+1)= & A x_{v a}(k)+B^{*} M_{v a} x_{v a}(k-1) \\
& +\left(B-B^{*}\right) u_{c}(k), \\
u_{f}(k)= & N_{v a}(\phi(k))\left(u_{c}(k)-M_{v a} x_{v a}(k-1)\right) \\
& -f_{a}(k),
\end{aligned}
$$

where $x_{v a} \in \mathbb{R}^{n_{x}}$ denotes the virtual actuator internal states. Moreover, $M_{v a} \in \mathbb{R}^{n_{u} \times n_{x}}$ is the delayed virtual actuator gain, which needs to be designed.

Similarly, in order to introduce the virtual sensor, let us consider the following matrices:

$$
\begin{aligned}
N_{v s}(\gamma(k)) & =C C_{f}(\gamma(k))^{\dagger}, \\
C^{*} & =N_{v s}(\gamma(k)) C_{f}(\gamma(k)) .
\end{aligned}
$$

Assumption 2: The pair $\left(E, C^{*}\right)$ is assumed to be observable and hence matrices $E$ and $C^{*}$ satisfy the rank condition

$$
\operatorname{rank}\left[\begin{array}{c}
E \\
C^{*}
\end{array}\right]=n_{x} .
$$

If Assumption 2 holds, there exist matrices $T_{s} \in \mathbb{R}^{n_{x} \times n_{x}}$ and $N_{s} \in \mathbb{R}^{n_{x} \times n_{y}}$ satisfying the following condition

$$
T_{s} E+N_{s} C^{*}=I_{n_{x}} .
$$

Definition 3 Virtual sensor for descriptor systems: Given the descriptor system subject to actuator and sensor faults in (1), the virtual sensor is defined in (27), as shown at the bottom of the next page, where $x_{v s} \in \mathbb{R}^{n_{x}}$ and $z_{v s} \in \mathbb{R}^{n_{x}}$ denote the vector of internal states and intermediate states of the virtual sensor. Moreover, $M_{v s} \in \mathbb{R}^{n_{x} \times n_{y}}$ is the virtual sensor gain to be designed.

Remark 3: According to [27], matrices $B^{*}$ and $C^{*}$ are independent to faults $\phi(k)$ and $\gamma(k)$.

\section{Overall Analysis and Design}

Let us analyze the closed-loop dynamics of the augmented system composed of the faulty system (1), the delayed virtual actuator and the virtual sensor given by Definitions 2 and 3 as well as the nominal observer-based delayed controller (21).

Theorem 5: Consider the faulty descriptor system (1), the nominal controller in (21), the delayed virtual actuator in (23) and the virtual sensor (27). Let us define a new vector of state variables $\zeta(k)=\left[\zeta_{1}(k)^{\top}, \zeta_{2}(k)^{\top}, \zeta_{3}(k)^{\top}, \zeta_{4}(k)^{\top}\right]^{\top}$ with

$$
\begin{aligned}
& \zeta_{1}(k)=x_{v a}(k), \\
& \zeta_{2}(k)=x_{f}(k)+x_{v a}(k), \\
& \zeta_{3}(k)=\hat{x}(k)-x_{v s}(k), \\
& \zeta_{4}(k)=x_{v s}(k)-x_{f}(k)-x_{v a}(k) .
\end{aligned}
$$

Then, the dynamics of $\zeta$ is described by

$$
\tilde{E} \zeta(k+1)=\tilde{A} \zeta(k)+\tilde{A}_{d} \zeta(k-1),
$$

where $\tilde{E}, \tilde{A}$ and $\tilde{A}_{d}$ are defined in (30), as shown at the bottom of the next page, with $\Xi=\left(T A-L C^{*}\right)-\left(T E+N C^{*}\right)$ $\left(T_{s} A-M_{v s} C^{*}\right)$.
Proof: See Appendix A.

Based on Theorem 5 with the separation principle in Theorem 1, the admissibility of the closed-loop system in (29) is equivalent to admissibility of the following subsystems:

$$
\begin{aligned}
E \delta(k+1) & =A \delta(k)+B^{*} M_{v a} \delta(k-1), \\
E \delta(k+1) & =A \delta(k)+B K \delta(k-1), \\
\delta(k+1) & =(T A-L C) \delta(k), \\
\delta(k+1) & =\left(T_{s} A-M_{v s} C^{*}\right) \delta(k),
\end{aligned}
$$

where $\delta \in \mathbb{R}^{n_{x}}$ denotes an auxiliary state.

From (31a) and (31b), the design of the gains of the delayed virtual actuator and of the nominal controller correspond to the descriptor delay system form in (8). Thus, they can be designed using Theorem 4.

On the other hand, since (31c) and (31d) are in a non-descriptor form, the design of the gains of the state observer and of the virtual sensor can be performed using standard Lyapunov stability results, see e.g. [34].

\section{FTC Implementation Algorithm}

The implementation steps are summarized in Algorithm 1.

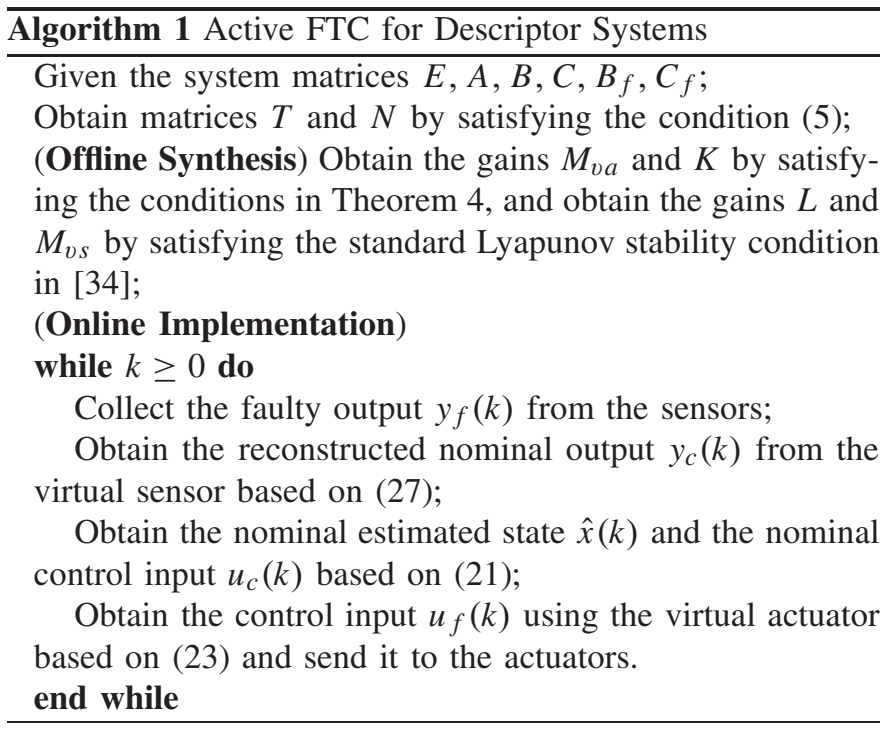

\section{Simulation Results}

\section{A. Example 1: Admissibility Analysis}

Let us consider the following first-order system with state delay

$$
x(k+1)=a x(k)+a_{d} x(k+1),
$$

with scalar parameters $a, a_{d}$, and let us analyze its admissibility according to the theoretical results provided in Section III-B. Taking into account that all matrices $A=a$, $A_{d}=a_{d}$ and $E=1$ are scalars, the conditions of Proposition 1 become

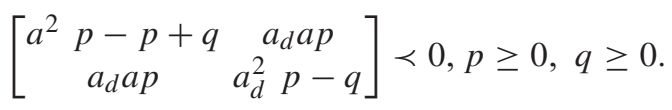




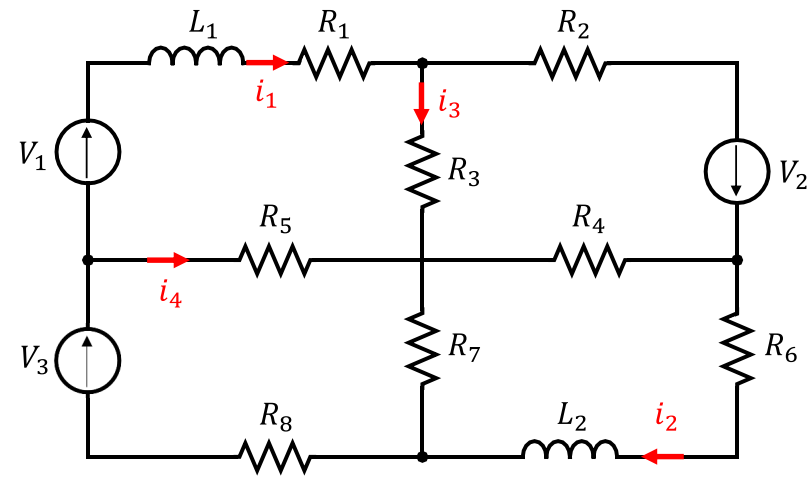

Fig. 3. Example 2: electrical circuit scheme.

In order to find out the conditions that the system's parameters $a$ and $a_{d}$ must satisfy to assess admissibility, let us consider the characteristic polynomial of the above matrix, which is

$$
\begin{aligned}
p(\lambda)= & \lambda^{2}+\lambda\left[p\left(1-a^{2}-a_{d}^{2}\right)\right] \\
& +\left(p-q-a^{2} p\right)\left(q-a_{d}^{2} p\right)-a_{d} a p .
\end{aligned}
$$

By applying the Descartes' rule of signs, a necessary condition for negativity of both roots of $p(\lambda)$ is $a^{2}+a_{d}^{2}<1$. Let us consider $a=-a_{d}=0.8$, which corresponds to $a^{2}+a_{d}^{2}=1.28$, so that the above necessary condition is not satisfied, which means that Proposition 1 would fail in assessing the admissibility. Nevertheless, Theorem 2 provides feasible conditions, since the following solution is returned by the MOSEK solver [35]: $P=1.17, S=0.72, Q=0.78$.

\section{B. Example 2: Application to an Electrical Circuit}

Consider the electrical circuit shown in Figure 3. Based on the Kirchoff's laws, the corresponding differential and algebraic equations are obtained as follows:

$$
\begin{aligned}
L_{1} \dot{i}_{1} & =-R_{1} i_{1}-R_{3} i_{3}+R_{5} i_{4}+V_{1}, \\
L_{2} \dot{i}_{2} & =\left(R_{4}+R_{7}\right) i_{1}-\left(R_{4}+R_{6}+R_{7}\right) i_{2}-R_{4} i_{3}+R_{7} i_{4}, \\
0 & =\left(R_{7}+R_{8}\right) i_{1}-R_{7} i_{2}+\left(R_{5}+R_{7}+R_{8}\right) i_{4}-V_{3}, \\
0 & =-\left(R_{2}+R_{4}\right) i_{1}+R_{4} i_{2}+\left(R_{2}+R_{3}+R_{4}\right) i_{3}+V_{2}, \\
y_{1} & =i_{1}+i_{4}, \\
y_{2} & =i_{1}-i_{3}, \\
y_{3} & =i_{2}-i_{1}-i_{4}, \\
y_{4} & =i_{4},
\end{aligned}
$$

where $L_{1}=0.3 H, L_{2}=0.65 H, R_{1}=10 \Omega, R_{2}=17 \Omega$, $R_{3}=3 \Omega, R_{4}=5 \Omega, R_{5}=2 \Omega, R_{6}=27 \Omega, R_{7}=8 \Omega$ and $R_{8}=10 \Omega$. Set the vectors $x=\left[\begin{array}{llll}i_{1} & i_{2} & i_{3} & i_{4}\end{array}\right]^{\top}$, $u=\left[\begin{array}{lll}V_{1} & V_{2} & V_{3}\end{array}\right]^{\top}$ and $y=\left[\begin{array}{llll}y_{1} & y_{2} & y_{3} & y_{4}\end{array}\right]^{\top}$. By applying an Euler discretization with sampling time $T_{s}=0.01 \mathrm{~s}$, a discretetime descriptor model as in (3) is obtained, with

$$
\begin{aligned}
& E=\left[\begin{array}{llll}
1 & 0 & 0 & 0 \\
0 & 1 & 0 & 0 \\
0 & 0 & 0 & 0 \\
0 & 0 & 0 & 0
\end{array}\right], A=\left[\begin{array}{cccc}
0.67 & 0 & -0.1 & 0.07 \\
0.2 & 0.38 & -0.08 & 0.12 \\
18 & -8 & 0 & 20 \\
-22 & 5 & 25 & 0
\end{array}\right], \\
& B=\left[\begin{array}{ccc}
0.03 & 0 & 0 \\
0 & 0 & 0 \\
0 & 0 & -1 \\
0 & 1 & 0
\end{array}\right], C=\left[\begin{array}{cccc}
1 & 0 & 0 & 1 \\
1 & 0 & -1 & 0 \\
-1 & 1 & 0 & 1 \\
0 & 0 & 0 & 1
\end{array}\right] .
\end{aligned}
$$

Let us consider the closed-loop system includes partial and total loss of actuators and sensors, that is, $B_{f}=$ $B \operatorname{diag}(0.8,0.4,0), C_{f}=\operatorname{diag}(0.8,0.2,0.7,0) C, f_{a}=0$ and $f_{s}=0$. These actuator and sensor faults are injected into the closed-loop system from the sampling step $k=8$.

Since the separation principle holds for the closed-loop FTC system as discussed in Theorem 1 and Theorem 5, we can design separately the nominal controller and observer, delayed virtual actuator and virtual sensor to obtain the gains as follows:

$$
\begin{aligned}
& K=\left[\begin{array}{cccc}
-6.8514 & -0.5272 & 0.4857 & -0.5038 \\
3.9852 & -0.9889 & 0.9712 & -0.7700 \\
2.5770 & -1.8868 & -0.0432 & -0.0585
\end{array}\right], \\
& L=\left[\begin{array}{l}
18.4571-24.9163-3.1442-1.5725 \\
18.4276-24.9279-2.9419-1.3125 \\
18.4571-24.9263-3.1442-1.5725 \\
17.8804-25.0163-3.1442-1.0525
\end{array}\right], \\
& M_{v a}=\left[\begin{array}{cccc}
-6.7414 & -0.4498 & 0.4362 & -0.4814 \\
1.4438 & -0.5625 & 1.0963 & -0.9369
\end{array}\right] \text {, } \\
& M_{v s}=\left[\begin{array}{cccc}
-86.9881 & 28.0430 & -2.7376 & 158.1273 \\
-39.3522 & 3.9590 & -2.4778 & 86.3636 \\
-87.0385 & 27.8508 & -2.7579 & 158.1629 \\
-33.8528 & 0.5126 & -3.0026 & 76.7430
\end{array}\right] \text {. }
\end{aligned}
$$

To test the proposed FTC strategy, we enable the FTC from the sampling step $k=15$. The closed-loop results are shown in Figure 4(a)-4(d). The results also include the comparison with the closed-loop trajectory without faults as well as with faults but without introducing FTC. For all the four states,

$$
\begin{aligned}
z_{v s}(k+1) & =\left(T_{s} A-M_{v s} C^{*}\right) x_{v s}(k)+T_{s} B u_{c}(k)+M_{v s} N_{v s}(\gamma(k))\left(y_{f}(k)+C_{f}(\gamma(k)) x_{v a}(k)-f_{s}(k)\right), \\
x_{v s}(k) & =z_{v s}(k)+N_{s} N_{v s}(\gamma(k))\left(y_{f}(k)+C_{f}(\gamma(k)) x_{v a}(k)-f_{s}(k)\right), \\
y_{c}(k) & =N_{v s}(\gamma(k))\left(y_{f}(k)+C_{f}(\gamma(k)) x_{v a}(k)-f_{s}(k)\right)+\left(C-C^{*}\right) x_{v s}(k) .
\end{aligned}
$$

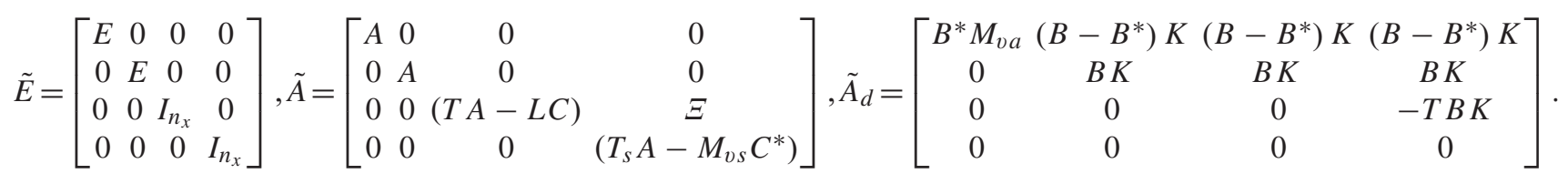




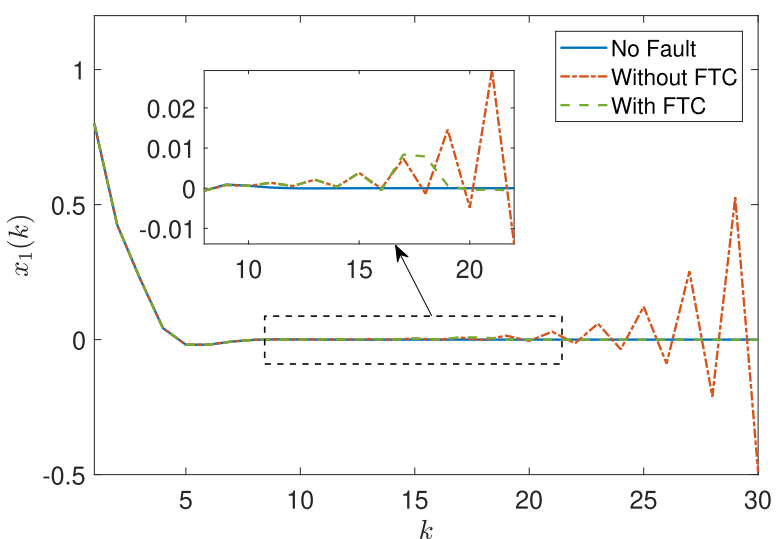

(a) $x_{1}$

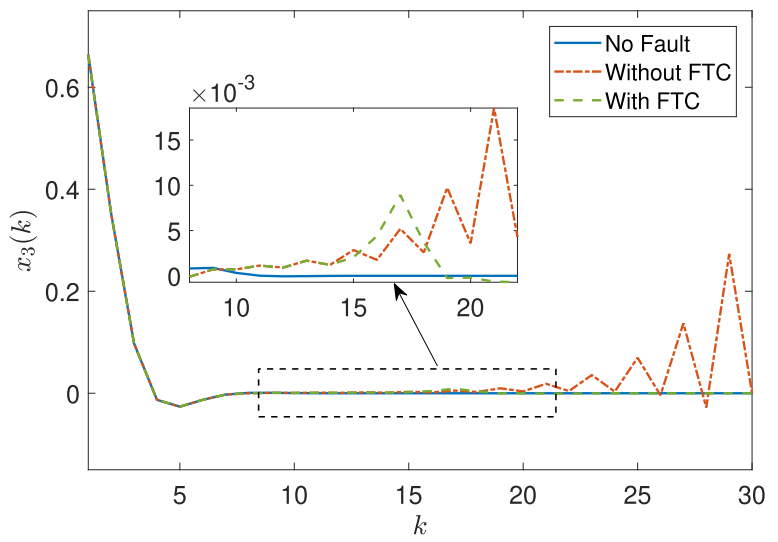

(c) $x_{3}$

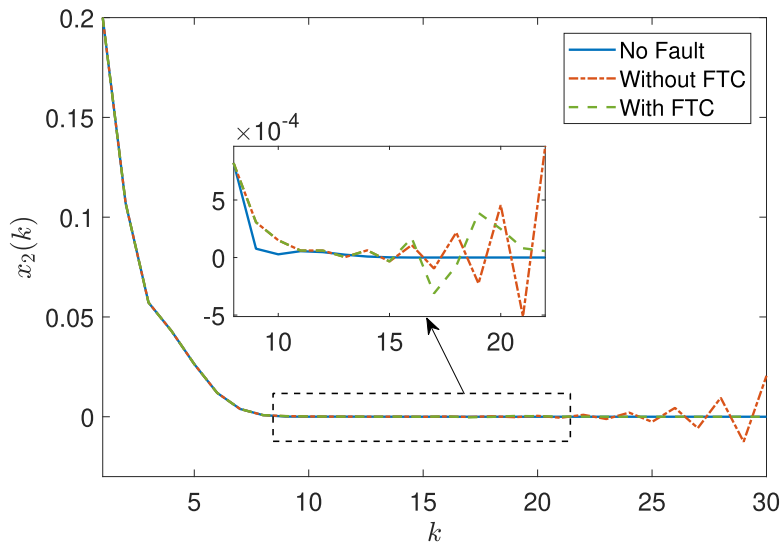

(b) $x_{2}$

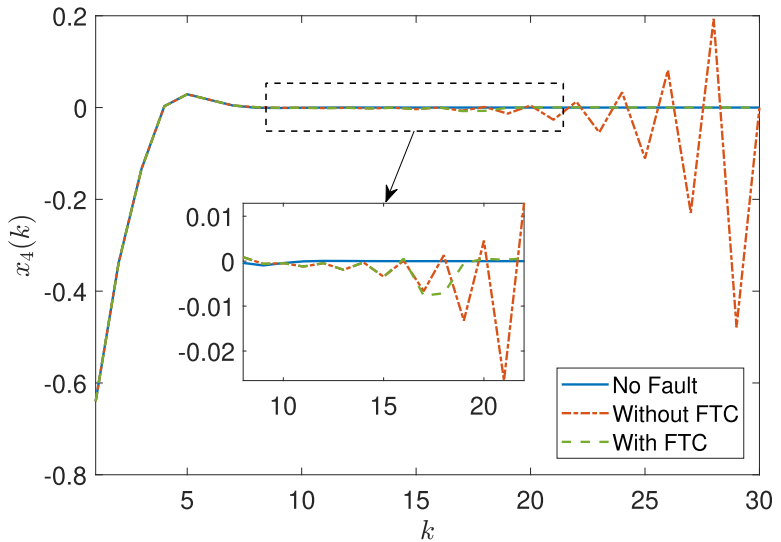

(d) $x_{4}$

Fig. 4. Example 2: comparison results with and without FTC.

when the faults occurred, the closed-loop system becomes unstable without FTC. When the proposed FTC is applied, the closed-loop system becomes stable again and converges to zero after a transient.

\section{Example 3: Scalability}

This section presents an extensive numerical validation of the design approach presented in this article, with the aim of illustrating its scalability to higher order systems. In particular, for different system orders between $n=5$ and $n=12$, 100 systems have been generated by assuming $r=\lceil n / 2\rceil$, with $E$ obtained as $E=\operatorname{diag}\left(I_{r}, 0_{n-r}\right)$, the availability of three inputs and three outputs $\left(n_{u}=3, n_{y}=3\right)$, and values for the elements of the matrices $A, B, C$ drawn from normal distributions, using a variance of 0.25 for the state matrix and 1 for the input and output matrices. For each of these systems, complete losses of the third actuator and the third sensor have been considered for the design of the corresponding virtual actuator/virtual sensor. The results in terms of percentage of systems for which feasibility of the design of all the components (controller, virtual actuator, observer, virtual sensor) was obtained are shown in Table I, along with the average time needed to solve the matrix inequalities (a laptop equipped with an Intel(R) Core(TM) i7-8565U CPU @ 1.80 GHz 1.99GhZ processor has been used). The results show that, although the
TABLE I

EXAMPLE 3: SCALABILITY WITH INCREASING SYSTEM ORDER,

\begin{tabular}{|c|c|c|c|c|c|}
\hline$n$ & $\%$ Feas. & Av. Time & $n$ & $\%$ Feas. & Av. Time \\
\hline 5 & $65 \%$ & $22.69 \mathrm{~s}$ & 6 & $61 \%$ & $47.55 \mathrm{~s}$ \\
\hline 7 & $58 \%$ & $55.74 \mathrm{~s}$ & 8 & $49 \%$ & $134.15 \mathrm{~s}$ \\
\hline 9 & $35 \%$ & $201.36 \mathrm{~s}$ & 10 & $26 \%$ & $368.18 \mathrm{~s}$ \\
\hline 11 & $24 \%$ & $550.98 \mathrm{~s}$ & 12 & $17 \%$ & $1024.8 \mathrm{~s}$ \\
\hline
\end{tabular}

performance of the approach and the average computation time get worse when the system order increases, it is still possible to apply the proposed approach for higher order systems. It must be remarked that computations are performed offline, such that the issues arising from the increase of needed time can be partially alleviated by the availability of more efficient computational equipment as well as the natural increase in computational power brought by technological advances.

\section{CONCLUSION}

This article has proposed an active FTC scheme for discrete-time descriptor systems. By means of the proposed VA and VS, the closed-loop system has been reconfigured so that fault-hiding has been achieved. For discrete-time descriptor systems, we have also proposed an observer-based feedback controller to deal with the algebraic loop in the implementation. For the synthesis of this feedback controller, 
we have proposed an improved admissibility condition, which has larger feasible region of solutions. Finally, we have applied the proposed active FTC scheme to several examples, among which an electrical circuit, showing that this FTC scheme is able to deal with partial and total loss of some actuators and sensors. Future work will aim at integrating the proposed design procedure with disturbance/noise rejection techniques that would increase the overall robustness of the FTC scheme.

\section{APPENDIX A \\ PROOF OF THEOREM 5}

From the definition of $\zeta(k)$ in $(28)$, the following relations can be obtained $x_{v a}(k)=\zeta_{1}(k), x_{f}(k)=-\zeta_{1}(k)+\zeta_{2}(k)$, $\hat{x}(k)=\zeta_{2}(k)+\zeta_{3}(k)+\zeta_{4}(k), x_{v s}(k)=\zeta_{2}(k)+\zeta_{4}(k)$.

From (23) and (21), it follows that

$$
\begin{aligned}
E \zeta_{1}(k+1)= & E x_{v a}(k+1) \\
= & A x_{v a}(k)+\left(B-B^{*}\right) K \hat{x}(k-1) \\
& +B^{*} M_{v a} x_{v a}(k-1) \\
= & A \zeta_{1}(k)+B^{*} M_{v a} \zeta_{1}(k-1) \\
& +\left(B-B^{*}\right) K \zeta_{2}(k-1) \\
& +\left(B-B^{*}\right) K \zeta_{3}(k-1) \\
& +\left(B-B^{*}\right) K \zeta_{4}(k-1) .
\end{aligned}
$$

From (1), (21) and (23), we have that

$$
\begin{aligned}
E \zeta_{2}(k+1)= & E x_{f}(k+1)+E x_{v a}(k+1) \\
= & A\left(x_{f}(k)+x_{v a}(k)\right)+B K \hat{x}(k-1) \\
= & A \zeta_{2}(k)+B K \zeta_{2}(k-1) \\
& +B K \zeta_{3}(k-1)+B K \zeta_{4}(k-1) .
\end{aligned}
$$

From (1), (23), (27) and (21), we have that

$$
\begin{aligned}
\zeta_{4}(k+1)= & x_{v s}(k+1)-x_{f}(k+1)-x_{v a}(k+1) \\
= & z_{v s}(k+1)+N_{s} C^{*}\left(x_{f}(k+1)+x_{v a}(k+1)\right) \\
& -x_{f}(k+1)-x_{v a}(k+1) .
\end{aligned}
$$

Taking into account the condition (26), the above equation can be simplified as

$$
\begin{aligned}
\zeta_{4}(k+1) & =z_{v s}(k+1)-T_{s} E\left(x_{f}(k+1)+x_{v a}(k+1)\right) \\
& =\left(T_{s} A-M_{v s} C^{*}\right)\left(x_{v s}(k)-x_{f}(k)-x_{v a}(k)\right) \\
& =\left(T_{s} A-M_{v s} C^{*}\right) \zeta_{4}(k) .
\end{aligned}
$$

From (1), (21) and (27), we have that

$$
\begin{aligned}
\zeta_{3}(k+1)= & \hat{x}(k+1)-x_{v s}(k+1) \\
= & (T A-L C) \hat{x}(k)+T B u_{c}(k) \\
& +L C^{*}\left(x_{f}(k)+x_{v a}(k)\right)+L\left(C-C^{*}\right) x_{v s}(k) \\
& +N C^{*}\left(x_{f}(k+1)+x_{v a}(k+1)\right) \\
& +N\left(C-C^{*}\right) x_{v s}(k+1) \\
& -\left(T_{s} A-M_{v s} C^{*}\right) x_{v s}(k)-T_{s} B u_{c}(k) \\
& -M_{v s} C^{*}\left(x_{f}(k)+x_{v a}(k)\right) \\
& -N_{s} C^{*}\left(x_{f}(k+1)+x_{v a}(k+1)\right) .
\end{aligned}
$$

Considering the conditions (5) and (26), the above equation becomes

$$
\begin{aligned}
\zeta_{3}(k+1)= & (T A-L C) \zeta_{3}(k)+\left(T A-L C^{*}\right) \zeta_{4}(k) \\
& \left.-\left(T E+N C^{*}\right)\left(T_{S} A-M_{v S} C^{*}\right)\right) \zeta_{4}(k) \\
& -T B K \zeta_{4}(k-1) .
\end{aligned}
$$

As a result, according to calculations above, it can be concluded that the closed-loop dynamics of $\zeta$ is given by (29).

\section{ACKNOWLEDGMENT}

The authors would like to thank the anonymous reviewers and the Associate Editor who handled this article for providing useful comments to improve the quality of this work.

\section{REFERENCES}

[1] G. Duan, Analysis and Design of Descriptor Linear Systems. New York, NY, USA: Springer, 2010.

[2] S. Xu and J. Lam, Robust Control and Filtering of Singular Systems. Berlin, Germany: Springer, 2006.

[3] A. Kumar and P. Daoutidis, Control of Nonlinear Differential Algebraic Equation Systems With Applications to Chemical Processes, vol. 397. Boca Raton, FL, USA: CRC Press, 1999.

[4] D. G. Luenberger and A. Arbel, "Singular dynamic Leontief systems," Econometrica J. Econ. Soc., vol. 5, no. 4, pp. 991-995, 1977.

[5] Q. Zhang, C. Liu, and X. Zhang, Complexity, Analysis and Control of Singular Biological Systems, vol. 421. London, U.K.: Springer, 2012.

[6] Y. Wang, V. Puig, and G. Cembrano, "Non-linear economic model predictive control of water distribution networks," J. Process Control, vol. 56, pp. 23-34, Aug. 2017.

[7] I. Masubuchi, J. Kato, M. Saeki, and A. Ohara, "Gain-scheduled controller design based on descriptor representation of LPV systems: Application to flight vehicle control," in Proc. 43rd IEEE Conf. Decis. Control (CDC), vol. 1, Dec. 2004, pp. 815-820

[8] L. Vermeiren, A. Dequidt, M. Afroun, and T.-M. Guerra, "Motion control of planar parallel robot using the fuzzy descriptor system approach," ISA Trans., vol. 51, no. 5, pp. 596-608, Sep. 2012.

[9] M. Blanke, M. Kinnaert, J. Lunze, M. Staroswiecki, and J. Schröder, Diagnosis Fault-Tolerant Control, vol. 2, 2nd ed. Berlin, Germany: Springer, 2006.

[10] Z. Zhou, M. Zhong, and Y. Wang, "Fault diagnosis observer and fault-tolerant control design for unmanned surface vehicles in network environments," IEEE Access, vol. 7, pp. 173694-173702, 2019.

[11] X. Zhang, X. Feng, Z. Mu, and Y. Wang, "State and fault estimation for nonlinear recurrent neural network systems: Experimental testing on a three-tank system," Can. J. Chem. Eng., vol. 98, no. 6, pp. 1328-1338, Jun. 2020.

[12] Y. Zhang and J. Jiang, "Bibliographical review on reconfigurable fault-tolerant control systems," Annu. Rev. Control, vol. 32, no. 2 , pp. 229-252, Dec. 2008.

[13] J. D. Stefanovski, "Passive fault tolerant perfect tracking with additive faults," Automatica, vol. 87, pp. 432-436, Jan. 2018.

[14] B. Rabaoui, M. Rodrigues, H. Hamdi, and N. B. Braiek, "A model reference tracking based on an active fault tolerant control for LPV systems," Int. J. Adapt. Control Signal Process., vol. 32, no. 6, pp. 839-857, Jun. 2018.

[15] Y. Wang, V. Puig, and G. Cembrano, "Robust fault estimation based on zonotopic Kalman filter for discrete-time descriptor systems," Int. J. Robust Nonlinear Control, vol. 28, no. 16, pp. 5071-5086, Nov. 2018.

[16] Y. Wang, S. Olaru, G. Valmorbida, V. Puig, and G. Cembrano, "Set-invariance characterizations of discrete-time descriptor systems with application to active mode detection," Automatica, vol. 107 pp. 255-263, Sep. 2019.

[17] J. D. Stefanovski, "Fault tolerant control of descriptor systems with disturbances," IEEE Trans. Autom. Control, vol. 64, no. 3, pp. 976-988, Mar. 2019.

[18] Y. Mu, H. Zhang, H. Su, and K. Zhang, "Observer-based actuator fault estimation and proportional derivative fault tolerant contro for continuous-time singular systems," Optim. Control Appl. Methods, vol. 40, no. 6, pp. 979-997, Nov. 2019. 
[19] J. Zhang, S. Li, and Z. Xiang, "Adaptive fuzzy output feedback eventtriggered control for a class of switched nonlinear systems with sensor failures," IEEE Trans. Circuits Syst. I, Reg. Papers, early access, May 27, 2020, doi: 10.1109/TCSI.2020.2994547.

[20] J. Lunze and T. Steffen, "Control reconfiguration after actuator failures using disturbance decoupling methods," IEEE Trans. Autom. Control, vol. 51, no. 10, pp. 1590-1601, Oct. 2006.

[21] G. Valencia-Palomo, F.-R. López-Estrada, and D. Rotondo, "Recent advances on optimization for control, observation, and safety," Processes, vol. 8, no. 2, p. 201, 2020.

[22] M. Yadegar, N. Meskin, and A. Afshar, "Fault-tolerant control of linear systems using adaptive virtual actuator," Int. J. Control, vol. 92, no. 8, pp. 1729-1741, Aug. 2019.

[23] J. H. Richter, W. P. M. H. Heemels, N. van de Wouw, and J. Lunze, "Reconfigurable control of piecewise affine systems with actuator and sensor faults: Stability and tracking," Automatica, vol. 47, no. 4, pp. 678-691, Apr. 2011.

[24] J. H. Richter, Reconfigurable Control of Nonlinear Dynamical Systems: A Fault-Hiding Approach, vol. 408. Berlin, Germany: Springer, 2011.

[25] D. Rotondo, F. Nejjari, and V. Puig, "Fault tolerant control of a proton exchange membrane fuel cell using Takagi-Sugeno virtual actuators," J. Process Control, vol. 45, pp. 12-29, Sep. 2016.

[26] R. Nazari, M. M. Seron, and J. A. De Doná, "Fault-tolerant control of systems with convex polytopic linear parameter varying model uncertainty using virtual-sensor-based controller reconfiguration," Аnnu. Rev. Control, vol. 37, no. 1, pp. 146-153, Apr. 2013.

[27] D. Rotondo, F. Nejjari, and V. Puig, "A virtual actuator and sensor approach for fault tolerant control of LPV systems," J. Process Control, vol. 24, no. 3, pp. 203-222, Mar. 2014.

[28] D. Rotondo, H. S. Sánchez, V. Puig, T. Escobet, and J. Quevedo, "A virtual actuator approach for the secure control of networked LPV systems under pulse-width modulated DoS attacks," Neurocomputing, vol. 365, pp. 21-30, Nov. 2019.

[29] M. Darouach, "Observers and observer-based control for descriptor systems revisited," IEEE Trans. Autom. Control, vol. 59, no. 5, pp. 1367-1373, May 2014.

[30] Z. Wang, Y. Shen, X. Zhang, and Q. Wang, "Observer design for discrete-time descriptor systems: An LMI approach," Syst. Control Lett., vol. 61, no. 6, pp. 683-687, Jun. 2012.

[31] B. Zhang, S. Xu, and Y. Zou, "Improved stability criterion and its applications in delayed controller design for discrete-time systems," Automatica, vol. 44, no. 11, pp. 2963-2967, Nov. 2008.

[32] K.-L. Hsiung and L. Lee, "Lyapunov inequality and bounded real lemma for discrete-time descriptor systems," IEE Proc.-Control Theory Appl., vol. 146, no. 4, pp. 327-331, Jul. 1999.

[33] S. Xu and J. Lam, "Robust stability and stabilization of discrete singular systems: An equivalent characterization," IEEE Trans. Autom. Control, vol. 49, no. 4, pp. 568-574, Apr. 2004.

[34] G. Duan and H. Yu, LMIs in Control Systems: Analysis, Design and Applications. Boca Raton, FL, USA: CRC Press, 2013.

[35] MOSEK ApS. (2015). The MOSEK Optimization Toolbox for MATLAB Manual Version 7.1 (Revision 28). [Online]. Available: http://docs.mosek.com/7.1/toolbox/index.html

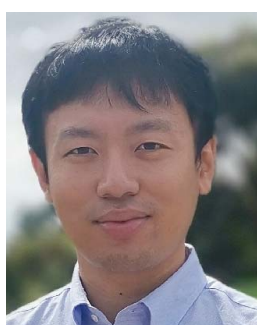

Ye Wang received the M.Sc. degree in automatic control and robotics and the Ph.D. degree (cum laude) in automatic control, robotics, and vision from the Universitat Politècnica de CatalunyaBarcelonaTech (UPC), Spain, in 2014 and 2018, respectively. From 2015 to 2018, he was a Research Fellow of the Spanish National Research Council (CSIC) at the Institut de Robòtica i Informàtica Industrial (IRI), CSIC-UPC. In February 2019, he joined the College of Intelligent Systems Science and Engineering, Harbin Engineering University, China, as an Associate Professor. Since September 2019, he has been a Research Fellow with the Department of Electrical and Electronic Engineering, University of Melbourne, Australia. His current research interest includes distributed control and optimization with applications to autonomous systems and cyber-physical systems. In 2019, he received the Best Ph.D. Thesis Award in control engineering from the Spanish National Committee of Automatic Control (CEA) and Springer.

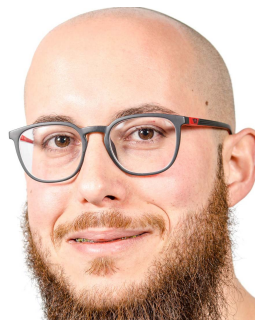

Damiano Rotondo received the B.S. degree (Hons.) from the Second University of Naples, Italy, in 2008, the M.Sc. degree (Hons.) from the University of Pisa, Italy, in 2011, and the Ph.D. degree (Hons.) from the Universitat Politècnica de Catalunya-BarcelonaTech (UPC), Spain, in 2016. Since February 2020, he has been an Associate Professor with the Department of Electrical Engineering and Computer Science (IDE), Universitetet i Stavanger (UiS), Norway. His main research interests include gain-scheduled control systems, fault detection and isolation (FDI), and fault-tolerant control (FTC) of dynamic systems.

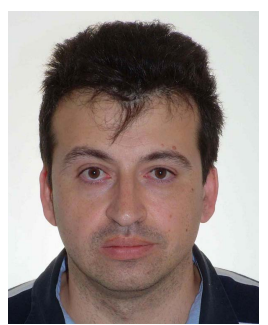

Vicenç Puig received the B.Sc. and M.Sc. degrees in telecommunications engineering and the Ph.D. degree in automatic control, vision, and robotics from the Universitat Politècnica de Catalunya-BarcelonaTech (UPC), Barcelona, Spain, in 1993 and 1999, respectively. He is currently a Full Professor with the Automatic Control Department, UPC, and a Researcher with the Institut de Robòtica i Informàtica Industrial (IRI), CSIC-UPC. He is also the Director of the Automatic Control Department and the Head of the Research Group on Advanced Control Systems (SAC), UPC. He has developed important scientific contributions in the areas of fault diagnosis and fault-tolerant control, using interval, and linear-parameter-varying models using set-based approaches. He has participated in more than 20 European and national research projects in the last decade. He has also led many private contracts with several companies and has published more than 220 journal articles as well as over 500 contributions in international conference/workshop proceedings. He has supervised over $25 \mathrm{Ph} . \mathrm{D}$. dissertations and over 50 master theses/final projects. He was the General Chair of the third IEEE Conference on Control and Fault-Tolerant Systems (SysTol 2016) and the IPC Chair of IFAC Safeprocess 2018. He is also the Chair of the IFAC Safeprocess TC Committee 6.4.

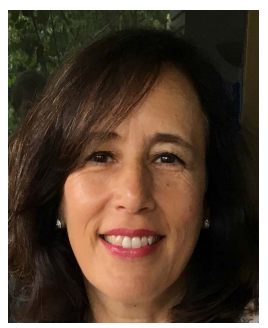

Gabriela Cembrano received the M.Sc. degree in industrial engineering and the Ph.D. degree in automatic control from the Universitat Politècnica de Catalunya-BarcelonaTech (UPC). She is a tenured Researcher of the Spanish National Research Council (CSIC) at the Institut de Robòtica i Informàtica Industrial (IRI), CSIC-UPC. Since 2007, she has been a Scientific Advisor of the CETaqua, Water Technology Centre. Her main research interest includes control engineering and its applications to water systems management. She has taken part in numerous fundamental and industrial research projects in this field since 1990. Most recently, she has been the Scientific Director of the EC Project EFFINET and the CSIC Leader in projects LIFE-EFFIDRAIN and DEOCS. She has published over 150 journal and conference papers in this field. 